\title{
A new schematic for poly(3- alkylthiophene) in an amorphous film studied using a novel structural index in infrared spectroscopy
}

\section{$\operatorname{AUTHOR}(S)$ :}

Shioya, Nobutaka; Shimoaka, Takafumi; Eda, Kazuo; Hasegawa, Takeshi

\section{CITATION:}

Shioya, Nobutaka ...[et al]. A new schematic for poly(3-alkylthiophene) in an amorphous film studied using a novel structural index in infrared spectroscopy. Physical Chemistry Chemical Physics 2015, 17(20): 13472-13479

\section{ISSUE DATE:}

2015

URL:

http://hdl.handle.net/2433/218638

\section{RIGHT:}

This is the accepted version of the following article, which has been published in final form at http://doi.org/10.1039/C5CP01024A; This is not the published version. Please cite only the published version.; この論文は出版社版でありません。引用の際には出版社版 をご確認ご利用ください。 
A New Schematic of Poly(3-alkylthiophene) in an Amorphous Film Studied by Using a Novel Structural Index on Infrared Spectroscopy

N. Shioya ${ }^{\mathrm{a}}$, T. Shimoaka ${ }^{\mathrm{a}}$, K. Eda ${ }^{\mathrm{b}}$, and T. Hasegawa ${ }^{\mathrm{a} *}$

Table of Contents

\section{Amorphous film}

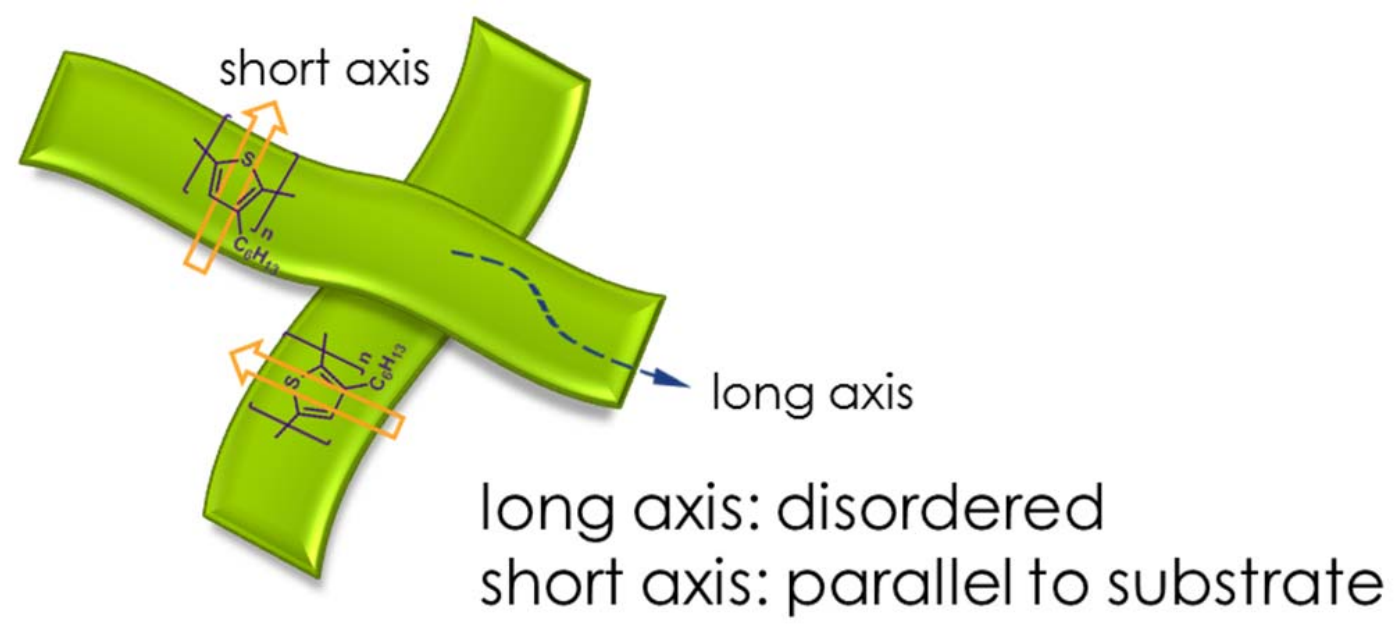


Abstract: The molecular structure of poly(3-alkylthiophene-2,5-diyl) (P3AT) in an amorphous film are revealed that the short axis of the thiophene ring is highly kept parallel to the substrate; whereas the long axis along the polymer chain is largely disordered, which is unveiled by infrared p-polarized multiple-angle incidence resolution spectrometry (pMAIRS) technique. This analysis is achieved by analyzing the orientation angles of mutually orthogonal three vibrational modes localized on the thiophene ring with an aid of a newly developed structural index. This new analytical technique is useful irrespective of the crystallinity of the thin film. As a result, the intrinsic chemical parameters controlling the molecular orientation are understood in a unified manner, and the reason the hexyl group gives the best result for a photovoltaic cell is also revealed. (127 words) 


\section{INTRODUCTION}

Poly(3-alkylthiophene-2,5-diyl) ${ }^{1}$ (P3AT) is a representative conjugated polymer compound having a solution processability, especially for the hexyl group-containing polythiophene, poly(3-hexylthiophene-2,5-diyl) (P3HT), which is employed as an active material in an organic field effect transistor $(\mathrm{OFET})^{2}$ and organic photovoltaic (OPV $)^{3}$ device due to the high carrier mobility in a thin film. ${ }^{4,5}$

The device performance strongly depends on the molecular aggregation structure represented by the molecular orientation and the crystallinity in the thin film. ${ }^{4,6-11}$ For example, when the thiophene rings are oriented parallel to the substrate in the film (the "face-on" orientation), the film is suitable for the active layer in $\mathrm{OPV},{ }^{7,8}$ since the charge transport is in the perpendicular direction to the substrate. On the other hand, in OFET, the source-drain electrodes are aligned parallel to the substrate. Considering this current direction, the thiophene stacking direction should be parallel to the substrate, in which the ring plane is oriented perpendicular to the substrate, corresponding to the "edge-on" orientation. ${ }^{4}$

A number of former studies report that the molecular orientation in the P3AT films can be controlled by the coating technique, ${ }^{4,11-14}$ the solvent, ${ }^{9,15}$ the substrate surface $^{16-18}$ and the material properties such as the molecular weight, ${ }^{18,19}$ the 
regioregularity ${ }^{4,14,20}$ and the chemical structure. ${ }^{10,21}$ For example, the face-on orientated P3AT film is obtained by: i) the spin-coating technique from chloroform solution ${ }^{22-24}$ and ii) the friction transferred method. ${ }^{11-12}$ On the other hand, the edge-on orientation is induced by: i) drop-casting ${ }^{4,14}$ or spin-coating at a low spin-speed, ${ }^{25}$ ii) using the high-boiling point solvents such as 1,2,4-trichlorobenzene ${ }^{9,24}$ and iii) drop-casting on a hydrophobic $\mathrm{Si}$ wafer surface covered with a self-assembled monolayer of hexamethyldisilazane or octadecyltrichlorosilane. ${ }^{16,18}$ The degree of orientation is further driven by the molecular weight, ${ }^{18-19}$ the regioregularity ${ }^{4,14,20}$ and the film thickness. ${ }^{14}$ In addition, the "end-on" orientation, in which the main chain has a perpendicular stance to the substrate, is generated by exposing a poly(3-buthylthiophene-2,5-diyl) (P3BT) film to carbon disulfide vapor. ${ }^{26}$ Also, Tajima and co-workers have recently reported that an end-functionalized $\mathrm{P} 3 \mathrm{BT}^{27}$ with a perfluoroundecyl group exhibits the end-on orientation in a spin-coated film due to the surface segregation of the perfluoroalkyl chains. ${ }^{21}$

In this manner, chemical factors controlling the molecular orientation in a P3AT film have extensively been investigated thus far. The correlation between the primary molecular structure and the final molecular orientation in a thin film is, however, still ambiguous and experiences are needed. For instance, the molecular orientation as a 
function of the alkyl sidechain length is not systematically revealed, although the length is known to significantly influence the thermal behavior ${ }^{28}$ and the device performance $^{29-31}$ via the conjugate length ${ }^{32}$ etc. The molecular mechanism, in addition, to explain the reason the "hexyl" chain yields a best result for both OPV and OFET devices is also still unclear.

One of the reasons of the ambiguous understanding is because an analytical technique using a laboratory equipment for revealing the molecular orientation of each chemical group is not recognized. To evaluate the molecular orientation in a P3AT film with a high sensitivity, the X-ray diffraction ${ }^{4,14}$ or near-edge X-ray absorption fine structure (NEXAFS) $)^{25,33}$ technique using a synchrotron radiation source is often employed, which is a barrier for analyzing a thin film in handy.

To get over the difficulty, IR pMAIRS ${ }^{34-37}$ that is a useful spectroscopic tool for revealing the molecular orientation in an organic thin film in a laboratory ${ }^{38,39}$ is powerful, since IR pMAIRS works irrespective of the crystallinity of the thin film. Since the polymer thin film prepared via a solution process often has a poor crystallinity due to the flexible long polymer chains, IR pMAIRS is useful to reveal the molecular orientation in a polymer thin film. ${ }^{24,40-42}$

In the present study, in addition, a novel structural index is proposed to make the 
better use of the IR pMAIRS technique to reveal the molecular orientation in a poor-crystallinity thin film. With the new technique, an accurate image of P3AT in an amorphous film has experimentally been revealed that the short axis of the thiophene ring proves to be highly kept parallel to the substrate surface while the long axis is largely disordered because of the poor alignment of the polymer chains. This technique is also highly useful to discriminate the ring-parallel orientation from the ring-vertical one on a Fourier transform infrared (FT-IR) spectrometer that is quite popular in a laboratory. As a result, the molecular orientation has been found to be strongly influenced by the molecular aggregation structure in the solution needed for the film preparation. In other words, the controlling mechanism of the molecular orientation considering the primary chemical structure via the molecular aggregation property has apparently been revealed by using the IR pMAIRS technique.

\section{EXPERIMENTAL METHODS:}

Sample preparations: Regioregular-poly(3-butylthiophene-2,5-diyl) (RR-P3BT) was obtained from Alfa Aesar (Ward Hill, MA, USA). RR-poly(3-hexylthiophene-2,5-diyl) (P3HT) $\left(M_{\mathrm{n}}: 15,000 \sim 45,000\right)$, poly(3-octylthiophene -2,5-diyl) (P3OT) $\left(M_{\mathrm{n}}: \sim 25,000\right)$ and poly(3-dodecylthiophene-2,5-diyl) (P3DDT) $\left(M_{\mathrm{n}}:\right.$ 27,000) were purchased from Aldrich (Milwaukee, WI, USA), which were used without further purification. 
Chloroform (Chl) of ACS Spectra Grade ( $\geq 99.8 \%$ ) was purchased from Sigma-Aldrich (St. Louis, MO, USA). P3ATs were dissolved in Chl with a concentration of $5.0-10.0$ $\mathrm{mg} \mathrm{mL} \mathrm{m}^{-1}$. The solution was heated at $70^{\circ} \mathrm{C}$ for $20 \mathrm{~min}$ to make the compounds fully dissolved in the solvent, since P3BT is particularly difficult to be dissolved in $\mathrm{Chl}^{26}{ }^{26}$ and the solution was left at room temperature over days. Once the compounds are dissolved, no precipitates appeared in the solution stored at the ambient temperature. These Films were prepared by the spin-coating technique at $8000 \mathrm{rpm}$ from the Chl solution on a silicon substrate with no thermal annealing. The film thickness was estimated by X-ray reflectivity to be $30 \pm 10 \mathrm{~nm}$. The substrate was a double-sided polished silicon wafer provided by Valqua FFT (Tokyo, Japan), with a thickness of $0.675 \pm 0.025 \mathrm{~mm}$, and no chemical surface treatment such as surface oxidization was performed.

IR MAIRS measurements: IR pMAIRS spectra were measured on a Thermo Fischer Scientific (Madison, WI, USA) Nicolet 6700 FT-IR spectrometer equipped with a Thermo Fisher Scientific (Yokohama, Japan) automatic MAIRS equipment (TN10-1500). The p-polarized IR ray for the pMAIRS measurements was obtained by a Harrick PWG-U1R wire-grid polarizer. The modulation frequency of the IR ray was 60 $\mathrm{kHz}$, which was detected by a liquid-nitrogen cooled MCT detector. The wavenumber resolution was $4 \mathrm{~cm}^{-1}$. The angle of incidence was changed from $9^{\circ}$ through $44^{\circ}$ by $5^{\circ}$ 
steps after experimental optimization. The number of accumulations of the interferogram was 1000 for each angle of incidence.

IR pMAIRS yields both IP (in-plane) and OP (out-of-plane) spectra simultaneously from an identical sample, which correspond to the conventional transmission and reflection-absorption spectra, respectively. The molecular orientation can directly be analyzed by choosing the optimal angles of incidence, ${ }^{37}$ with which no knowledge of optical parameters is needed. The orientation angle, $\phi$, is calculated by using the MAIRS dichroic ratio, $I_{\mathrm{IP}} / I_{\mathrm{OP}}$, as follows. ${ }^{39}$

$$
\phi=\tan ^{-1} \sqrt{\frac{2 I_{\mathrm{IP}}}{I_{\mathrm{OP}}}}
$$

Here, $I_{\mathrm{IP}}$ and $I_{\mathrm{OP}}$ are absorbances of an analytical band appeared in the IP and OP spectra, respectively, and $\phi$ is the uniaxial orientation angle of the vibrational mode defined from the surface normal. The simple calculation procedure of the molecular orientation is another benefit of using the IR pMAIRS technique.

UV-vis measurements: The UV-vis spectra were measured by the use of a Jasco (Tokyo, Japan) V-630 UV-vis spectrometer. The spin-coated films for the UV-vis analysis were prepared on $\mathrm{CaF}_{2}$ substrate, which was purchased from Pier Optics (Gunma, Japan), and the size of the substrate was $40 \times 20 \times 1 \mathrm{~mm}^{3}$.

XRD measurements: X-ray diffraction (XRD) analysis was performed on a Rigaku 
(Tokyo, Japan) Smartlab X-ray diffractometer by a parallel beam method, using $\mathrm{Cu}-\mathrm{K} \alpha$ radiation $(\lambda=0.154 \mathrm{~nm})$ generated at $40 \mathrm{kV}$ and $30 \mathrm{~mA}$. The angle between the incidental X-ray and the substrate was fixed at $0.15^{\circ}$, which is smaller than the critical angle of the total reflection at the air/silicon interface $\left(0.23^{\circ}\right)$.

\section{RESULTS AND DISCUSSION}

Molecular orientation of the thiophene rings: Figure 1 through 4 present IR pMAIRS spectra of four P3AT films: P3BT, P3HT, P3OT and P3DDT prepared on a silicon wafer. The red and blue spectra are the MAIRS-IP and OP spectra, respectively. In the fingerprint region, the ring anti-symmetric stretching (denoted as $\mathrm{v}(\mathrm{C}=\mathrm{C})$ ) and $\mathrm{C}-\mathrm{H}$ out-of-plane deformation vibration $(\gamma(\mathrm{C}-\mathrm{H}))$ modes of the thiophene ring appear at ca. 1510 and $820 \mathrm{~cm}^{-1}$, respectively. ${ }^{43}$ Although the mode at ca. $820 \mathrm{~cm}^{-1}$ is denoted as $\delta(\mathrm{C}-$ $\mathrm{H})$ in our previous study, ${ }^{24}$ this mode is rewritten as $\gamma(\mathrm{C}-\mathrm{H})$ to emphasize that it is the 'out-of-plane' deformation vibration in the present study. In former studies, the $\gamma(\mathrm{C}-\mathrm{H})$ mode is found highly localized at the $\mathrm{C}-\mathrm{H}$ group of the thiophene ring, which has a perpendicular transition moment to the ring plane, ${ }^{24,44}$ whereas the $v(C=C)$ mode is highly oriented along the polymer skeletal direction. ${ }^{33,44}$ Fortunately, the absorption coefficients of these modes are large enough to analyze the molecular orientation in a thin film. Therefore, the $\gamma(\mathrm{C}-\mathrm{H})$ and $v(\mathrm{C}=\mathrm{C})$ bands are both quite useful for discussing 
the orientation of the thiophene ring ${ }^{11,13,24,44}$ and the polymer skeleton ${ }^{33,44}$ in a thin film (Figure 5).

The $v(\mathrm{C}=\mathrm{C})$ band at ca. $1510 \mathrm{~cm}^{-1}$ has a common trend to both P3BT and P3HT films in Figures 1 and 2, respectively: the IP band is apparently larger than the OP one. This MAIRS dichroic ratio (Eq. 1) indicates that the main chain should lie on the substrate surface in both films. At this moment, the ring-parallel and ring-vertical orientations are indistinguishable, and a further molecular orientation analysis of the thiophene ring is necessary to determine the orientation. For the purpose, the $\gamma(\mathrm{C}-\mathrm{H})$ band works powerfully. This band exhibits a largely different dichroic ratio between the P3BT and P3HT films. While the P3BT film has a stronger IP band at ca. $820 \mathrm{~cm}^{-1}$ than the OP one (Figure 1), the P3HT one has a dominantly strong OP band (Figure 2). This indicates that the P3BT and P3HT films can be categorized into the 'ring-vertical' and 'ring-parallel' orientations, respectively. ${ }^{24}$ The molecular orientation in a P3AT film thus sensitively responds to the alkyl sidechain length. In a similar manner, the molecular orientation in the P3OT and P3DDT films was analyzed (Figure 3 and 4). Both films are categorized into the ring-parallel orientation, which is a common trend to the P3HT film.

With the IR pMAIRS spectra, the orientation angles, $\phi_{\mathrm{C}-\mathrm{H}}$ and $\phi_{\mathrm{C}=\mathrm{C}}$, are easily 
calculated by using Eq. $1 .{ }^{39}$ The $\phi_{\mathrm{C}-\mathrm{H}}$ is calculated by the use of the $\gamma(\mathrm{C}-\mathrm{H})$ band, which is the angle between the phenyl ring-normal and the substrate surface-normal (Figure $5 \mathrm{~b}$ ). Of note is that the $\phi_{\mathrm{C}-\mathrm{H}}$ geometrically equals to the tilt angle, $\phi_{\text {tilt }}$, between the thiophene ring and the substrate surface (Figure 5b). On the other hand, the $\phi_{\mathrm{C}=\mathrm{C}}$ is calculated by the use of the $\mathrm{v}(\mathrm{C}=\mathrm{C})$ band, which corresponds to the angle between the polymer main chain and the surface normal (Figure 5b). Note that the calculated angle is the average of all the rings involved in the film.

The calculated orientation angles are listed in Table 1. The P3BT film, which has the ring-vertical orientation, has a larger $\phi_{\mathrm{C}=\mathrm{C}}\left(76^{\circ}\right)$ than the rest films $(\mathrm{P} 3 \mathrm{HT}, \mathrm{P} 3 \mathrm{OT}$ and P3DDT) having the ring-parallel orientation. This means that the main chains are better ordered in the film for the ring-vertical orientation, which corresponds to a fact that the P3BT film has a higher crystallinity than the ring-parallel films as discussed later.

When Table 1 is referred, the P3HT film has the smallest tilt angle of $\phi_{\mathrm{C}-\mathrm{H}}$ $\left(28^{\circ}\right)$ of the four samples. Therefore, the P3HT film is found to have architecture closest to the ring-parallel orientation in the P3AT family. When the attached alkyl chain is longer, the tilt angle becomes larger keeping the ring-parallel orientation.

A new schematic image of the face-on orientation: Here, the reason why the 
thiophene rings are tilted even in a "ring-parallel" film. When a polymeric molecule takes the ring-parallel orientation, the molecules should theoretically be piled up with a parallel orientation to the substrate surface. In practice, however, the long polymer chains cannot help being piled up in a messy manner, which makes the molecules disordered (Figure 5a). In fact, the tilt angle of the long axis of the thiophene ring ( $x$-axis in Figure $5 \mathrm{a}$ ), $\phi_{\mathrm{C}=\mathrm{C}}$, is close to the magic angle of $54.7^{\circ}$ especially for P3OT and P3DDT (Table 1).

What would happen, if the short axis of the ring (y-axis in Figure 5a) is fixed parallel to the substrate $\left(\phi_{y}=90^{\circ}\right)$ ? In this situation, $\phi_{\mathrm{C}-\mathrm{H}}(\mathrm{z}$-axis in Figure 5a) depends on only the x-axis. By considering $\phi_{\mathrm{C}-\mathrm{H}}=\phi_{\mathrm{tilt}}$ and the scheme in Figure 5b, the next relation holds:

$$
\phi_{x}+\phi_{z}=\phi_{\mathrm{C}=\mathrm{C}}+\phi_{\mathrm{C}-\mathrm{H}}=\phi_{\mathrm{C}=\mathrm{C}}+\phi_{\mathrm{tilt}}=90^{\circ}
$$

In fact, P3HT, P3OT and P3DDT films roughly satisfies this theoretical expectation: $\phi_{\mathrm{C}=\mathrm{C}}+\phi_{\mathrm{C}-\mathrm{H}}$ falls in a range from $83^{\circ}$ to $91^{\circ}$ (Table 1 ), which paradoxically implies that the short axis of the thiophene ring is highly kept parallel to the substrate, which is an accurate schematic of the ring-parallel orientation. In this manner, $\phi_{\mathrm{C}=\mathrm{C}}+\phi_{\mathrm{C}-\mathrm{H}}$, is a quite useful index to reveal the structural characteristic of the ring-parallel orientation of the long polythiophene. 
When the molecules are spontaneously aggregated (not piled up) via the inter-plane interaction of the thiophene rings to form a "planar aggregate" on the substrate, on the other hand, the short axis of the polymer chain should stand perpendicularly to the substrate surface. In short, the ring-vertical orientation is induced by the molecular spontaneous aggregation, in which the index of $\phi_{\mathrm{C}=\mathrm{C}}+\phi_{\mathrm{C}-\mathrm{H}}$ should be largely apart from $90^{\circ}$, since the short axis is far from parallel to the substrate surface. In fact, the P3BT film is true of this 'edge-on' orientation pattern (Table $1 ; \phi_{\mathrm{C}=\mathrm{C}}+$ $\left.\phi_{\mathrm{C}-\mathrm{H}}=135^{\circ}\right)$. In this manner, the new index, $\phi_{\mathrm{C}=\mathrm{C}}+\phi_{\mathrm{C}-\mathrm{H}}$, proved to be an useful marker to distinguish the ring-parallel orientation from the ring-vertical one for the P3AT thin films. The molecular orientation image discussed by the orientation angle analysis in the previous section has thus been reconfirmed.

Molecular orientation analysis on the alkyl sidechain length: As discussed above, the orientation of the thiophene ring is not a monotonous function of the alkyl chain length: only P3BT is an outlier. To consider the influence of the alkyl sidechain length, the IR bands in the $\mathrm{C}-\mathrm{H}$ stretching vibration region $\left(3000-2800 \mathrm{~cm}^{-1}\right)$ are analyzed. In Figures 1-4, both IP and OP spectra have a similar shape and intensity, which apparently implies that the alkyl chains are disordered in the film. ${ }^{24}$ In fact, the $\mathrm{CH}_{2}$ anti-symmetric stretching vibration $\left(\mathrm{v}_{\mathrm{a}}\left(\mathrm{CH}_{2}\right)\right)$ band, which is known as a 
conformation-sensitive marker, ${ }^{45,46}$ is apparently higher shifted $\left(2922-2928 \mathrm{~cm}^{-1}\right)$ than the all-trans position (ca. $\left.2917 \mathrm{~cm}^{-1}\right) .{ }^{46}$

In general, the conformation improves as the alkyl chain length increases due to the van der Waals interaction between the alkyl chains. ${ }^{47}$ Nevertheless, even in P3DDT with the long alkyl sidechains, the spectra indicate that the dodecyl chains are not ordered (Figure 4). In fact, the $\mathrm{CH}_{2}$ symmetric stretching vibration $\left(\mathrm{v}_{\mathrm{s}}\left(\mathrm{CH}_{2}\right)\right)$ band also exhibits a higher wavenumber than the all-trans position $\left(2850 \mathrm{~cm}^{-1}\right){ }^{46}$

Of another importance is that the $v_{a}\left(\mathrm{CH}_{2}\right)$ band in the OP spectra of the P3BT, P3OT and P3DDT films exhibits a slight high-wavenumber shift (MAIRS-shift) as compared to that in the IP spectrum (Figure 1, 3 and 4); whereas the P3HT film exhibits no MAIRS-shift (Figure 2). According to Hasegawa et al., ${ }^{48,49}$ the MAIRS-shift is a useful marker that 1) the alkyl chain has a largely disordered (nearly bending) conformation and 2) the entire chain has a roughly perpendicular stance to the substrate surface. Since the P3BT film has the ring-vertical orientation, the second item is satisfied. Therefore, the MAIRS-shift in Figure 1 should be attributed to the poor molecular aggregation between the too short alkyl chains.

On the other hand, the P3OT and P3DDT films also exhibit the MAIRS-shift although both films have the ring-parallel orientation, which strongly suggests that a 
disordered conformation of the alkyl chains due to the long sidechain is due to the bent chains "out of the film plane." In this sense, only the exception of the P3HT film is impressive: no MAIRS-shift strongly implies that the hexyl chain has an appropriate length that can be involved within a molecular plane, although the conformation is disordered $\left(v_{\mathrm{a}}\left(\mathrm{CH}_{2}\right)\right.$ at $\left.2927 \mathrm{~cm}^{-1}\right)$. This unique planar molecular aggregate structure with the ring-parallel orientation should be the reason why P3HT is chosen for a practical OPV device, which works for smooth charge transportation perpendicular to the substrate.

Confirmation of the molecular aggregation in films: The ring-vertical orientation is generated as a result of a strong inter-molecular interaction, which should be correlated with the crystallinity. To confirm this expectation, XRD patterns of the four P3AT films were measured as presented in Figure 6. As expected, only the P3BT film exhibits a strong diffraction peak at a low angle $\left(2 \theta=7.2^{\circ}\right)$, which is correlated with the index of 100 associated with the crystallographic direction along the alkyl sidechains in the lamellar structure. ${ }^{50}$ On the other hand, the rest P3HT, P3OT and P3DDT films have ambiguously weak diffraction peaks only, which indicates that these three films have an extremely low crystallinity i.e., nearly amorphous state. This straightforwardly implies that the ring-parallel orientation is not driven by the $\pi$-stacking or formation of the 
lamellar structure, which agrees with the IR results.

The difference in molecular aggregation should also be reflected in UV-vis spectra. Figure 7 shows two representative UV-vis spectra of the P3BT and P3HT in chloroform solution (a) and in films (b). According to Clark et al., an UV-vis spectrum of P3HT consists of short and long wavelength components. ${ }^{51}$ The short wavelength component is correlated with the amorphous regions having disordered chains; whereas the long wavelength one is due to the crystallite regions, in which the thiophene rings are weakly associated with each other via the H-aggregation. In addition, Brown et al. states that the shoulder peak at $610 \mathrm{~nm}$ in the long wavelength component is a result of the inter-chain $\pi-\pi$ interaction. ${ }^{52}$ It is apparent in Figure $7 \mathrm{~b}$ that the P3BT "film" has the long wavelength component compared to the P3HT film, and thus P3BT in the film proves to have a stronger molecular aggregation property than P3HT. This result supports the XRD analysis. The molecular aggregation property influences the molecular orientation in a film via the solubility in a solution as discussed below.

Influence of the solubility on the molecular orientation: The UV-vis spectrum of the chloroform "solution" of P3BT (blue curve in Figure 7a) apparently has two long-wavelength components at $560 \mathrm{~nm}$ and $610 \mathrm{~nm}$ as found in the film spectrum (Figure $7 \mathrm{~b}$ ). This implies that the molecular aggregate is generated even in a P3BT 
"solution." In other words, when the molecules are aggregated in the solution for preparing the film, the aggregates would remain in the film to induce the ring-vertical orientation. The aggregation property in a solution should be related to the solubility in an organic solvent. In fact, P3BT has a poor solubility in an organic solvent ${ }^{26}$ due to the short alkyl sidechains.

In a similar manner, UV-vis spectra of a chloroform solution and a film of P3HT were measured (Figure 7). The red spectrum of the P3HT "solution" exhibits a shorter-wavelength shift to that of the "film." The shoulder component at $560 \mathrm{~nm}$ recognized in the film spectrum is completely lost in the solution spectrum. Thus, the difference of the chain length of the butyl and hexyl chains makes the solubility largely different. In this manner, the solubility of P3AT is a conveniently useful factor to predict the final molecular orientation.

Influence of the zipper effect on the molecular orientation: On the other hand, the UV-vis spectra of P3AT except P3BT in the "solution" are nearly identical to each other (Figure 8a). The P3HT, P3OT and P3DDT "films", however, yield largely different spectra from each other: the main band exhibits a red shift and the shoulder peak at 610 $\mathrm{nm}$ increases with increasing the alkyl chain length (Figure 8b). This indicates that the intermolecular association improves with the alkyl chain length, which means that the 
P3HT film has the weakest molecular aggregation property of the three ring-parallel films. In fact, this correlates well with the IR result that P3HT most favors the ring-parallel orientation in the P3AT family.

According to Tashiro et al., ${ }^{32}$ the conjugation length of a thiophene skeletal chain measured at room temperature increases as the alkyl sidechain length increases due to the "zipper effect": the planar arrangement of the thiophene rings improves with an aid of the interaction between the neighboring alkyl sidechains. Considering this zipper effect, P3DDT with the long alkyl sidechain should have a strongest molecular aggregation property in the four samples, and P3BT is expected to have a weakest one. In fact, P3DDT has a larger intermolecular interaction than P3HT and P3OT in the film as evidenced by the intensity of the shoulder peak at $610 \mathrm{~nm}$ in the UV-vis spectra. P3BT has, however, another characteristic that the molecules are highly aggregated in the film reflecting the poor solubility in a solvent. As a result of the concerned solubility and zipper effects, P3HT becomes the winner to have the smallest molecular aggregation property to yield the "best ring-parallel" orientation in the P3AT family.

Summary: A novel structural index is proposed, which is useful to specify the ring-parallel orientation in a P3AT thin film by using the quantitative molecular orientation angles revealed by the IR pMAIRS technique. The new index of $\phi_{\mathrm{C}=\mathrm{C}}+$ 
$\phi_{\mathrm{C}-\mathrm{H}}$ is highly useful, which readily distinguishes the two different orientations: the ring-parallel orientation satisfies $\phi_{\mathrm{C}=\mathrm{C}}+\phi_{\mathrm{C}-\mathrm{H}} \approx 90^{\circ}$; whereas the summation is significantly greater than $90^{\circ}$ in the ring-vertical one. By using the structural index, the molecular orientation analysis of the P3AT films has become an easy task on a laboratory equipment of FT-IR. By using the chemically rich information of the IR spectra, the chemical mechanism to control the molecular orientation has been revealed as a function of the sidechain length of P3AT, which was readily supported by XRD and UV-vis data

P3BT has the ring-vertical orientation in the film, since $\phi_{\mathrm{C}=\mathrm{C}}+\phi_{\mathrm{C}-\mathrm{H}}\left(135^{\circ}\right)$ is significantly greater than $90^{\circ}$. The short sidechain of the butyl group particularly contributes to the strong molecular aggregation at an early stage in a solution, which has a strong correlation with the poor solubility in a solvent. As a result, the compound retains the aggregation character after transferred in a film, and the thiophene ring plane is oriented perpendicular to the substrate. This is the chemical origin of the ring-vertical orientation.

On the other hand, the P3HT, P3OT and P3DDT films roughly satisfy $\phi_{\mathrm{C}=\mathrm{C}}+\phi_{\mathrm{C}-\mathrm{H}}=90^{\circ}$, which indicates that these compounds have the ring-parallel orientation guaranteeing a highly parallel orientation of the short axis of the thiophene 
ring to the substrate surface. The P3HT film is, in addition, found to have the best ring-parallel orientation in the P3AT family because of a balance of the solubility and zipper effect.

Acknowledgment: This work was financially supported by a Grant-in-Aid for Young Scientists (B) (No. 26810075 (TS)) and Grant-in-Aid for Challenging Exploratory Research (No. 26620118 (TH)) from Japan Society for the Promotion of Science, from Japan Society for the Promotion of Science.

Notes and references

Corresponding Author

E-mail: htakeshi@scl.kyoto-u.ac.jp

FAX: $\quad+81774383074$

${ }^{a}$ Laboratory of Solution and Interface Chemistry, Division of Environmental Chemistry, Institute for Chemical Research, Kyoto University, Gokasho, Uji, Kyoto 611-0011, Japan

${ }^{b}$ Department of Chemistry, Graduate School of Science, Kobe University, 1-1 Rokko-dai, Nada-ku, Kobe, Hyogo 657-8501, Japan

1. M. Brinkmann, J. Polym. Sci., Part B: Polym. Phys., 2011, 49, 1218-1233.

2. J. Zaumseil and H. Sirringhaus, Chem. Rev., 2007, 107, 1296-1323. 
3. S. Günes, H. Neugebauer and N. S. Sariciftci, Chem. Rev., 2007, 107, 1324-1338.

4. H. Sirringhaus, P. Brown, R. Friend, M. M. Nielsen, K. Bechgaard, B. M. W. Langeveld-Voss, A. J. H. Spiering, R. A. J. Janssen, E. W. Meijer, P. Herwig and D. M. Leeuw, Nature, 1999, 401, 685-688.

5. Z. Bao, A. Dodabalapur and A. J. Lovinger, Appl. Phys. Lett., 1996, 69, 4108-4110.

6. C. Videlot, A. E. Kassmi and D. Fichou, Sol. Energy Mater. Sol. Cells, 2000, 63, 69-82.

7. I. Osaka, M. Shimawaki, H. Mori, I. Doi, E. Miyazaki, T. Koganezawa and K. Takimiya, J. Am. Chem. Soc. 2012, 134, 3498-3507.

8. X. Guo, N. Zhou, S. Lou, J. Smith, D. B. Tice, J. W. Hennek, R. P. Ortiz, J. T. L. Navarrete, S. Li, J. Strzalka, L. Chen, R. P. H. Chang, A. Facchetti and T. J. Marks, Nat. Photon., 2013, 7, 825-833.

9. J.-F. Chang, B. Sun, D. W. Breiby, M. M. Nielsen, T. I. Sölling, M. Giles, I. McCulloch and H. Sirringhaus, Chem. Mater., 2004, 16, 4772-4776.

10. J. Ma, K. Hashimoto, T. Koganezawa and K. Tajima, Chem. Commun., 2014, 50, 3627-3630.

11. T. Mizokuro, Y. Okamoto, C. Heck, H. Aota and N. Tanigaki, J. Apply. Polym. Sci. 2014, 131, 40136. 
12. S. Nagamatsu, W. Takashima, K. Kaneto, Y. Yoshida, N. Tanigaki, K. Yase and K. Omote, Macromolecules, 2003, 36, 5252-5257.

13. N. Tanigaki, S. Nagamatsu, W. Takashima and Y. Yoshida, Thin Solid Films, 2009, $518,853-856$

14. K. E. Aasmundtveit, E. J. Samuelsen, M. Guldstein, C. Steinsland, O. Flornes, C. Fagermo, T. M. Seeberg, L. A. A. Pettersson, O. Inganäs, R. Feidenhans'1 and S. Ferrer, Macromolecules, 2000, 33, 3120-3127.

15. P. K. -H. Ho, L. -L. Chua, M. Dipankar, X. Y. Gao, D. Qi, A. T. -S. Wee, J. -F. Chang and R. H. Friend, Adv. Mater., 2007, 19, 215-221.

16. W. Porzio, G. Scavia, L. Barba, G. Arrighetti and S. Milita, Eur. Polym. J., 2011, 47, 273-283.

17. S. Obata and Y. Shimoi, Phys. Chem. Chem. Phys., 2013, 15, 9265-9270.

18. R. J. Kline, M. D. McGehee and M. F. Toney, Nat. Mat., 2006, 5, 222-228.

19. R. J. Kline, M. D. McGehee, E. N. Kadnikova, J. Liu, J. M. J. Fréchet and M. F. Toney, Macromolecules, 2005, 38, 3312-3319.

20. R. D. McCullough, S. Tristram-Nagle, S. P. Williams, R. D. Lowe and M. Jayaraman, J. Am. Chem. Soc., 1993, 115, 4910-4911. 
21. J. Ma, K. Hashimoto, T. Koganezawa and K. Tajima, J. Am. Chem. Soc., 2013, 135, 9644-9647.

22. H. J. Fell, E. J. Samuelsen, J. Als-Nielsen, G. Grübel and J. Mårdalen, Solid State Commun., 1995, 94, 843-846.

23. K. E. Aasmundtveit, E. J. Samuelsen, J. Mrdalen, E. Bakken, P. H. J. Carlsen, and U. Lienert, Synth. Met., 1997, 89, 203-208.

24. N. Shioya, T. Shimoaka, and T. Hasegawa, Chem. Lett., 2014, 43, 1198-1200.

25. D. M. DeLongchamp, B. M. Vogel, Y. Jung, M. C. Gurau, C. A. Richter, O. A. Kirillov, J. Obrzut, D. A. Fischer, S. Sambasivan, L. J. Richter and E. K. Lin, Chem. Mater., 2005, 17, 5610-5612.

26. G. H. Lu, L. G. Li and X. N. Yang, Adv. Mater., 2007, 19, 3594-3598.

27. Y. Geng, K. Hashimoto and K. Tajima, Macromol. Chem. Phys., 2013, 214, $1326-1331$

28. V. Causin, C. Margea, A. Marigo, L. Valentini and J. M. Kenny, Macromolecules, 2005, 38, 409-415.

29. A. Babel and S. A. Jenekhe, Synth. Met., 2005, 148, 169-173.

30. L. H. Nguyen, H. Hoppe, T. Erb, S. Günes, G. Gobsch and N. S. Sariciftci, Adv. Funct. Mater., 2007, 17, 1071-1078. 
31. B. Friedel, C. R. McNeill and N. C. Greenham, Chem. Mater., 2010, 22, 3389-3398.

32. K. Tashiro, Y. Minagawa, M. Kobayashi, S. Morita, T. Kawai and K. Yoshino, Stnth. Met., 1993, 55-57, 321-328.

33. M. C. Gurau, D. M. Delongchamp, B. M. Vogel, E. K. Lin, D. A. Fischer, S. Sambasivan and L. J. Richter, Langmuir, 2007, 23, 834-842.

34. T. Hasegawa, J. Phys. Chem. B, 2002, 106, 4112-4115.

35. T. Hasegawa, L. Matsumoto, S. Kitamura, S. Amino, S. Katada and J. Nishijo, Anal. Chem., 2002, 74, 6049-6054.

36. T. Hasegawa, Anal. Chem., 2007, 79, 4385-4389.

37. T. Hasegawa, Y. Itoh and A. Kasuya, Anal. Sci., 2008, 24, 105-109.

38. T. Hasegawa, Anal. Bioanal. Chem., 2007, 388, 7-15.

39. T. Hasegawa, Appl. Spectrosc. Rev., 2008, 43, 181-201.

40. M. Matsunaga, T. Suzuki, K. Yamamoto and T. Hasegawa, Macromolecules, 2008, 41, 5780-5784.

41. H. Kakuda, T. Okada and T. Hasegawa, J. Phys. Chem. B, 2008, 112, 12940-12945.

42. Y. Nagao, J. Phys. Chem. C, 2013, 117, 3294-3297.

43. G. Louarn, M. Trznadel, J. Buisson, J. Laska, A. Pron, M. Lapkowski and S. Lefrant, J. Phys. Chem., 1996, 100, 12532-12539. 
44. S. Hotta, M. Soga and N. Sonoda, J. Phys. Chem., 1989, 93, 4994-4995.

45. R. G. Snyder, H. L. Strauss and C. A. Elliger, J. Phys. Chem., 1982, 86, 5145-5150.

46. R. A. MacPhail, H. L. Strauss, and R. G. Snyder, J. Phys. Chem., 1984, 88, 334-341.

47. M. Hostetler, J. Stokes and R. Murray, Langmuir, 1996, 12, 3604-3612.

48. T. Hasegawa, J. Umemura, C. Li and R. M. Leblanc, J. Phys. Chem. B, 2003, 107, 11996-12002.

49. T. Hasegawa, Y. Iiduka, H. Kakuda, and T. Okada, Anal. Chem., 2006, 78, $6121-6125$.

50. S. A. Chen and J. M. Ni, Macromolecules, 1992, 25, 6081-6089.

51. J. Clark, C. Silva, R. H. Friend and F. C. Spano, Phys. Rev. Lett., 2007, 98, 206406.

52. P. Brown, D. Thomas, A. Köhler, J. Wilson, J.-S. Kim, C. Ramsdale, H. Sirringhaus and R. Friend, Phys. Rev. B, 2003, 67, 064203. 
Table 1 Molecular orientation angles and the new index

\begin{tabular}{lcccc}
\hline \multicolumn{1}{c}{ P3AT } & $\phi_{\mathrm{C}-\mathrm{H}} /{ }^{\circ}$ & $\phi_{\mathrm{C}=\mathrm{C}} /{ }^{\circ}$ & $\phi_{\mathrm{C}-\mathrm{H}}+\phi_{\mathrm{C}=\mathrm{C}} /{ }^{\circ}$ & orientation \\
\hline P3BT & 59 & 76 & 135 & ring-vertical \\
P3HT & 28 & 63 & 91 & ring-parallel \\
P3OT & 30 & 53 & 83 & ring-parallel \\
P3DDT & 38 & 52 & 90 & ring-parallel \\
\hline
\end{tabular}




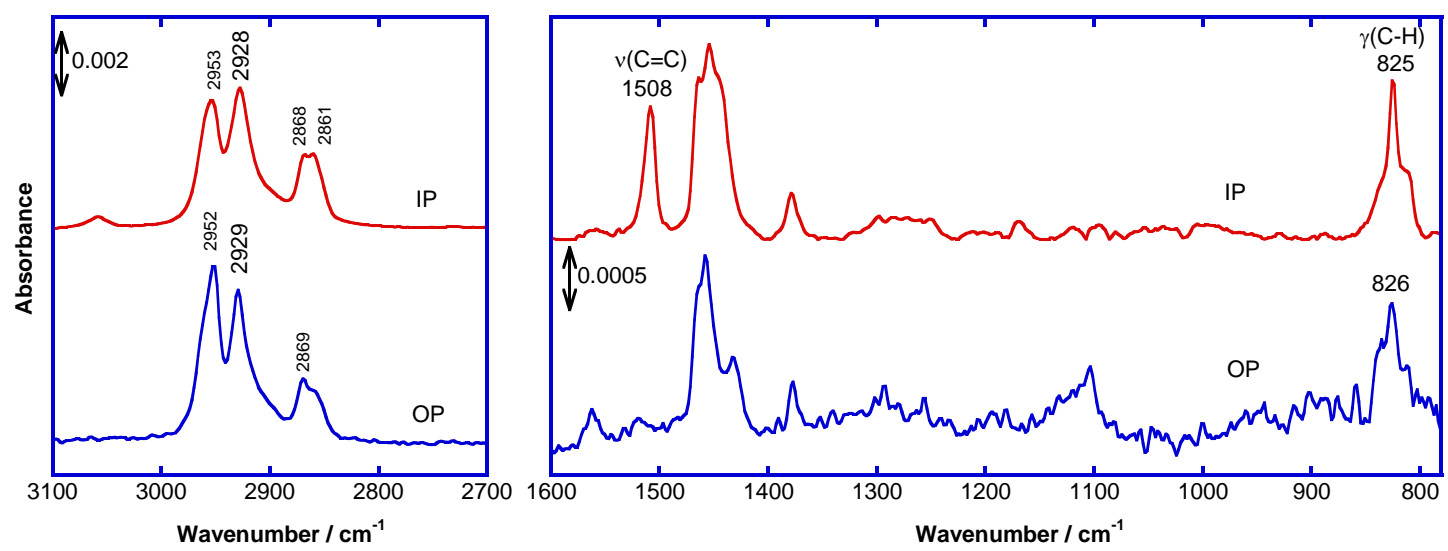

Figure 1 IR pMAIRS spectra of a thin film of P3BT on a Si substrate. 

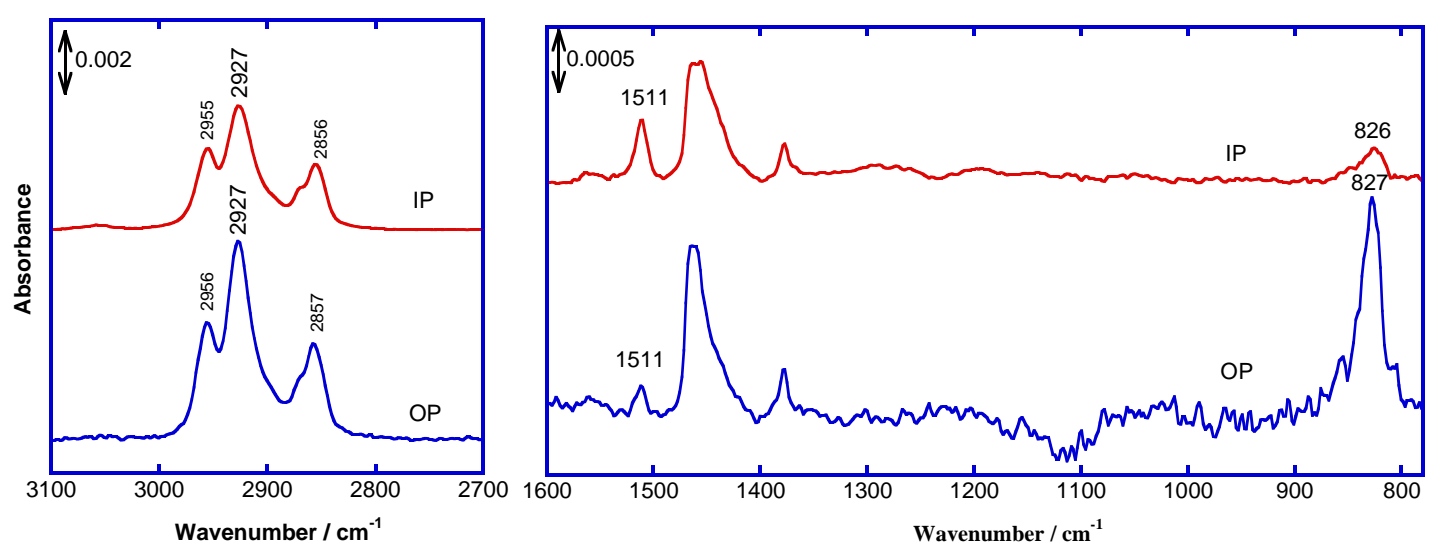

Figure 2 IR pMAIRS spectra of a thin film of P3HT on a Si substrate. 

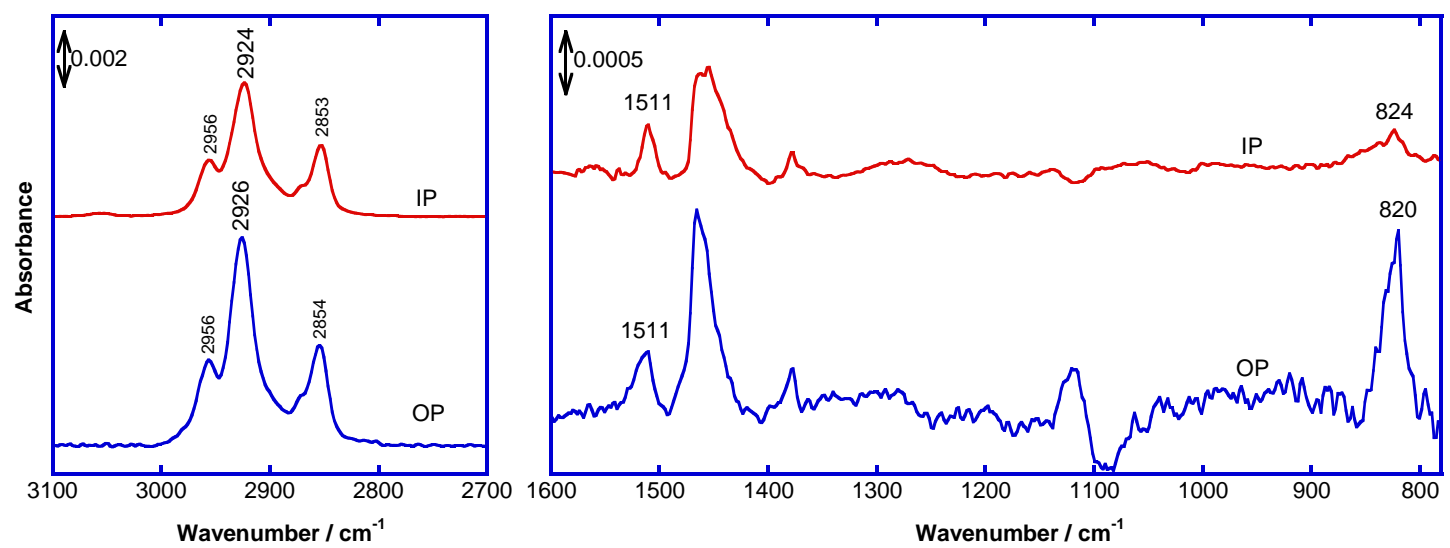

Figure 3 IR pMAIRS spectra of a thin film of P3OT on a Si substrate. 

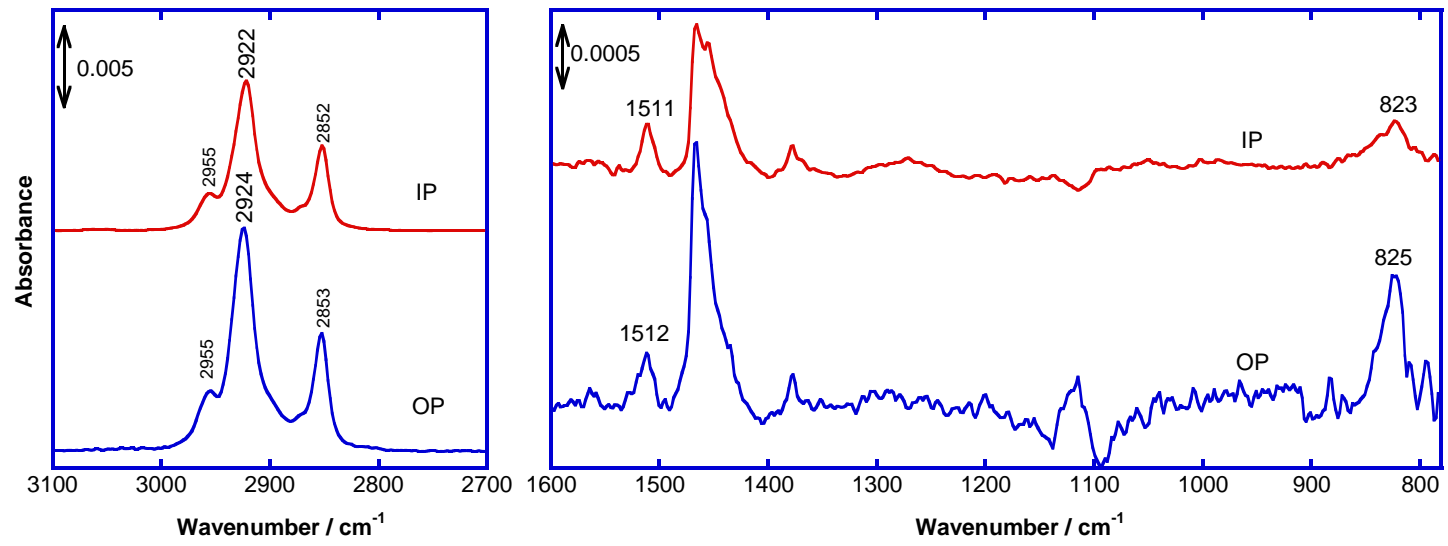

Figure 4 IR pMAIRS spectra of a thin film of P3DDT on a Si substrate. 
(a)

\section{ring-parallel}
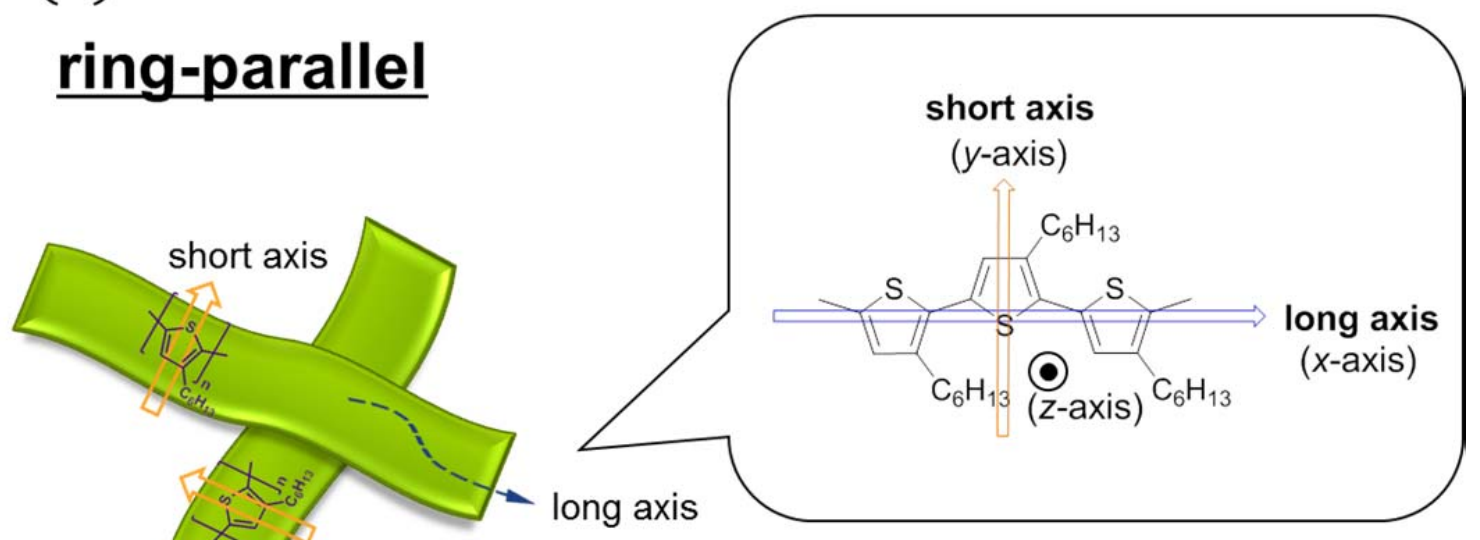

long axis: disordered

short axis: parallel to substrate

(b) ring-parallel (short axis || surface)

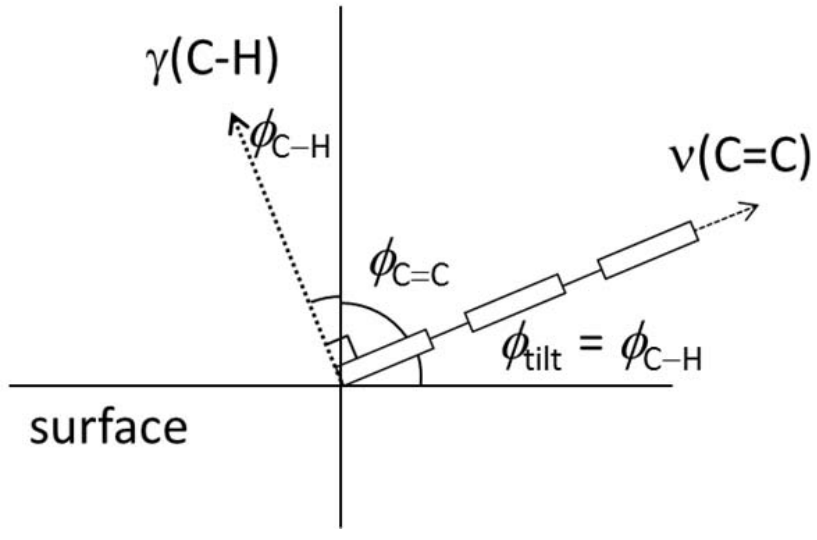

Figure 5 Schematic illustration of the overlaid polymer chains, and the newly introduced molecular coordinate ( $x, y$, and $z$-axes) fixed on a thiophene ring (a), and the interrelationship of the molecular orientation angles, $\boldsymbol{\phi}_{\mathbf{C}-\mathbf{H}}$ and $\boldsymbol{\phi}_{\mathbf{C}=\mathbf{C}}$. The tilt angle of the ring $\left(\phi_{\text {tilt }}\right)$ to the substrate equals to $\boldsymbol{\phi}_{\mathrm{C}-\mathrm{H}}$ when $\boldsymbol{\phi}_{\boldsymbol{y}}=\mathbf{9 0}^{\circ}$ (b), which results in $\phi_{\mathrm{C}=\mathrm{C}}+\phi_{\mathrm{C}-\mathrm{H}}=90^{\circ}$. 


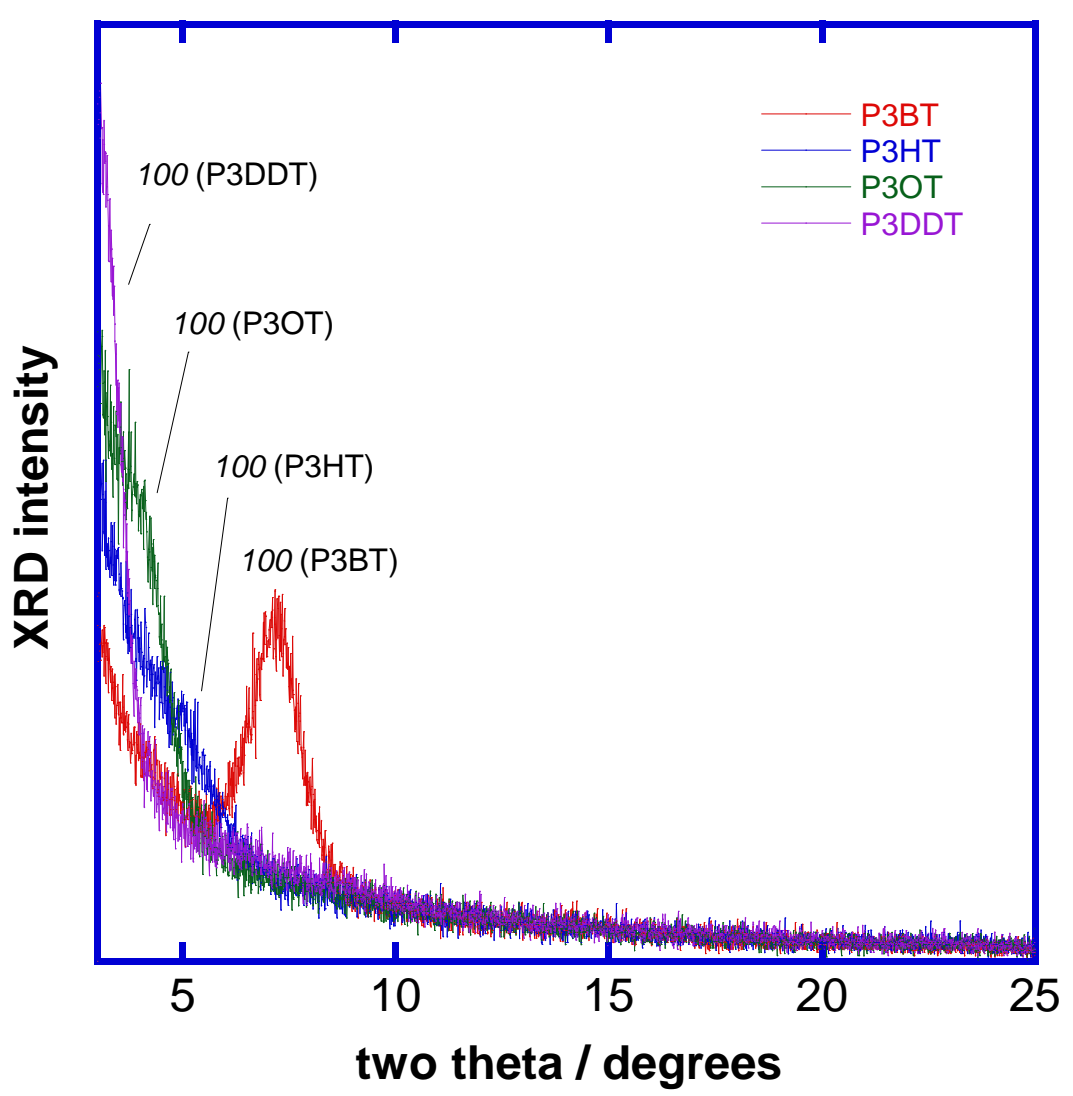

Figure 6 XRD patterns of the P3AT thin films on a Si substrate. 


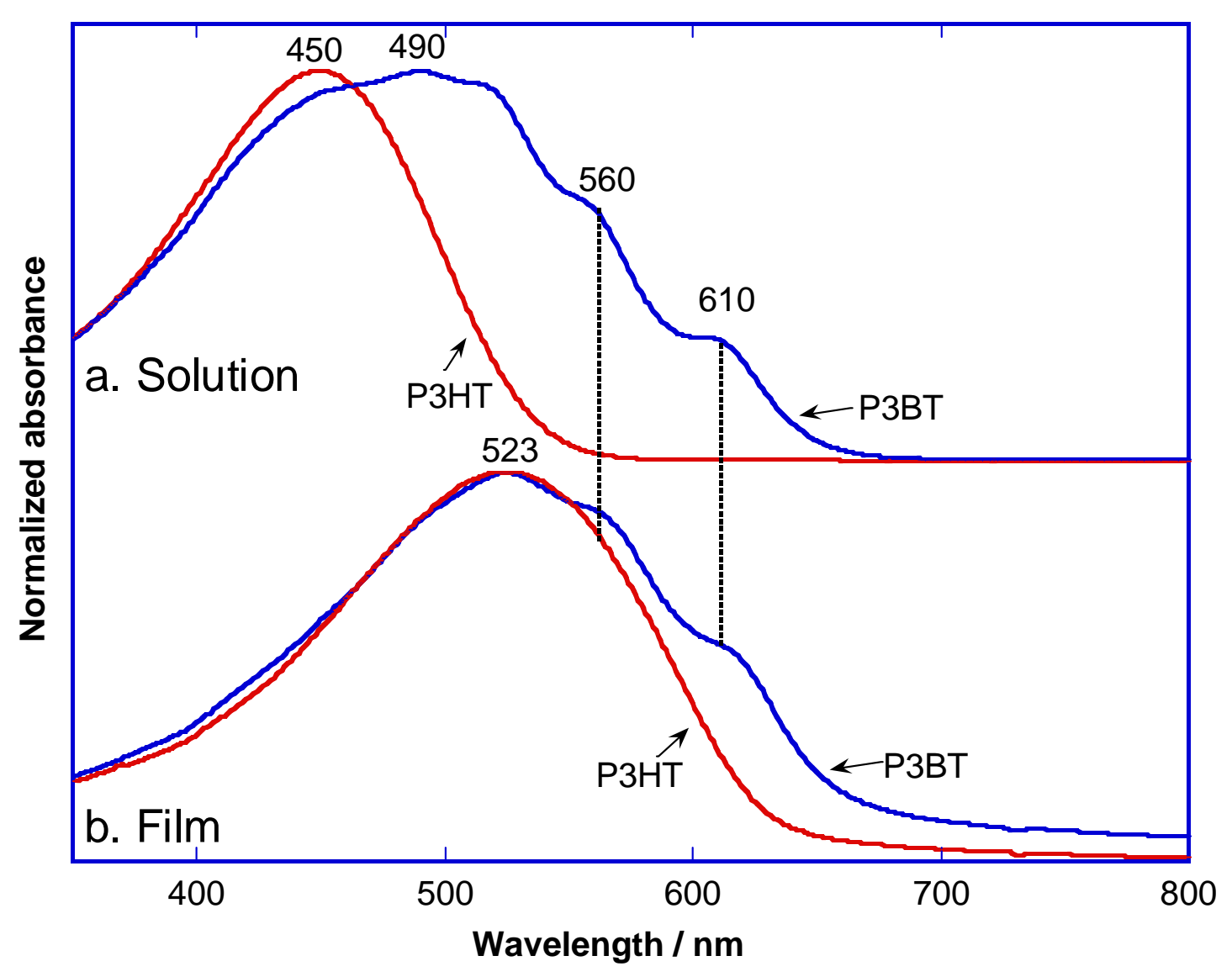

Figure 7 UV-vis spectra of P3BT and P3HT in a Chl solution (a), and in a thin film deposited on $\mathrm{a} \mathrm{CaF}_{2}$ substrate (b). 


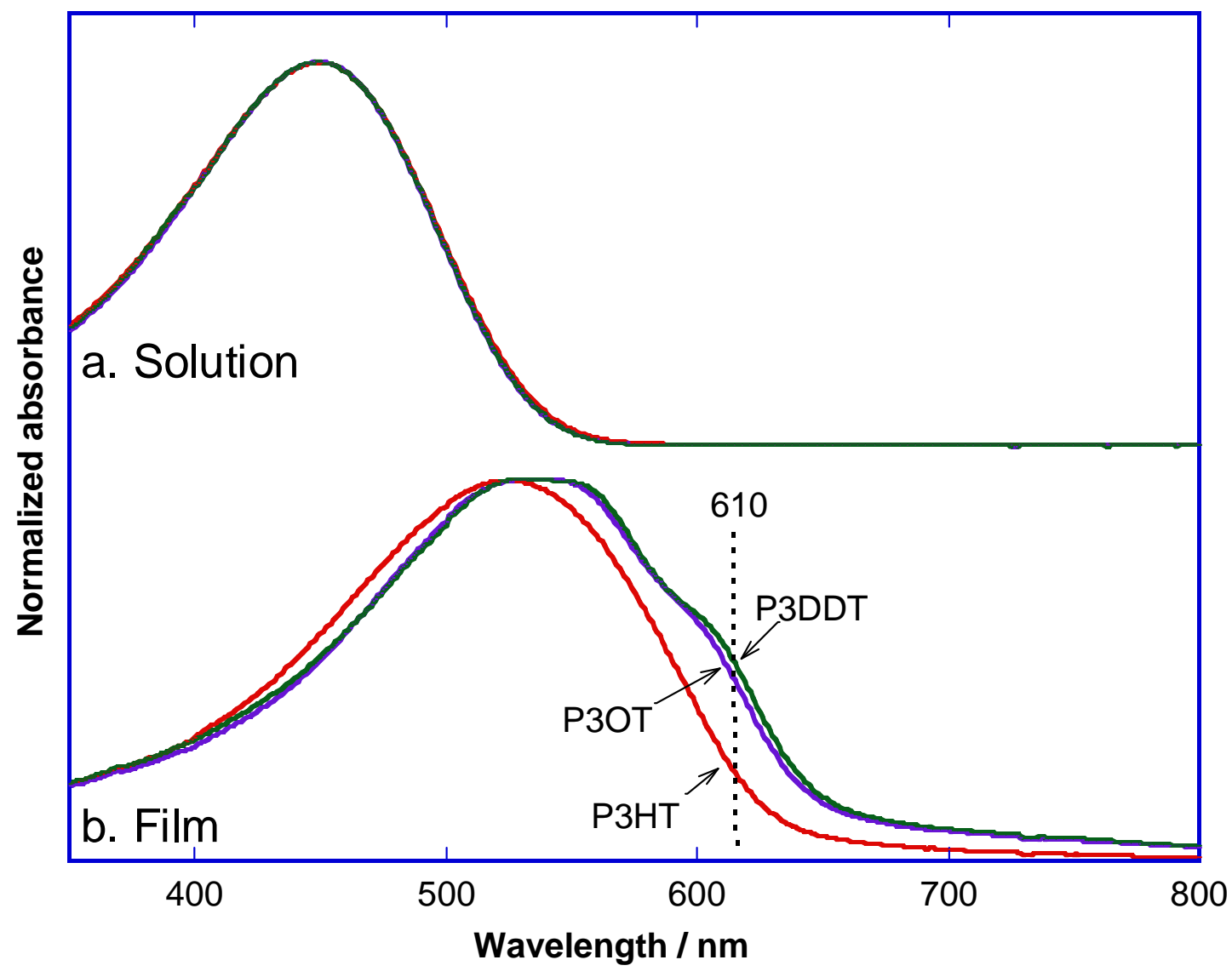

Figure 8 UV-vis spectra of P3HT, P3OT and P3DDT in a Chl solution (a), and in a thin film deposited on a $\mathrm{CaF}_{2}$ substrate (b). 